and epilepsy whose seizures are controlled with antiepileptic drugs. Pemoline (Cylert) is generally considered to have less tendency to lower seizure threshold than MPII. Some recommend an EEG in all ADDH patients considered for stimulant medications; those with a history of seizures and/or epileptiform discharges in the EEG should receive concomitant AED therapy. Children with ADDH have a $7 \%$ incidence of epileptiform EEGs. (Ped Neur Briefs Oct 1989; Progress in Pediatric

Neurology I, 1991, p 190).

\title{
COGNITIVE DEFICITS AND DEPRESSIVE SYMPTOMS
}

The efficacy of a new program (The Penn Prevention Program) to prevent depressive symptoms and alleviate associated deficits in academic achievement, peer relations, self-esteem, and behavior is reported in 69 'at risk' 10-13 year-old children evaluated in the Department of Psychology, University of Pennsylvania, Philadelphia, PA. Using cognitive-behavioral techniques proactively to teach coping strategies, children at risk of developing depression, based on level of symptoms and perception of parental conflict, showed significant reductions in depressive symptoms and improved classroom behavior. Benefits were sustained at 6-month follow-up, especially in children with higher risk factors, as compared to control groups. (Jaycox LH et al. Prevention of depressive symptoms in school children. Behav Res Ther Nov 1994;32:801-816). (Respond: Dr Lisa H Jaycox, Department of Psychology, University of Pennsylvania, 3815 Walnut Street, Philadelphia, PA 19104).

COMMENT. The authors hypothesize that children at risk for depression use skills learned in the Penn Prevention Program to deal with conflict and avoid depressive symptoms precipitated by interpersonal problems. This type of program could be important in children with ADDH and learning problems who are at increased risk of developing depressive symptoms associated with poor self esteem and academic failure.

\section{HEAD INJURY}

\section{POST-HEAD INJURY FUNCTIONAL DEFICITS}

The functional outcome in 95 children (aged 5 to 15 ) at 1 year after hospitalization for head injury was evaluated at the Johns Hopkins University, School of Hygiene and Public Health, Baltimore, MD. More than half of all injuries were motor vehicle related: $21 \%$ pedestrians, $18 \%$ passengers, and $17 \%$ bicycles. Lower extremity injuries were sustained in 20. Severity of head injury was determined using the Abbreviated Injury Scale (AIS 2-5) and the Glasgow Coma Scale (GCS 3-15). GCS were highly correlated with AIS severity. Chronic health problems pre-dating the injury were reported in 23\%; these were minor in $15 \%$, and major (mental retardation, seizures, lead poisoning) in $8 \%$. After controlling for head injury severity, poorer outcomes were associated with poverty, preinjury chronic health problems, and lower extremity injuries. At 1 year follow-up, $55 \%$ had one or more health problems: headaches in $32 \%$, limb or peripheral nerve disorders $(13 \%)$, weakness or ataxia $(7 \%)$, and vision, hearing, or speech disorder $(6 \%)$. The presence of functional limitations in physical activity (31\%) or self-care mobility (19\%) was associated especially with severe head injuries (AIS 5), but those with AIS 2-4 were not spared at least one limitation. Hyperactive behavior was directly correlated with head injury severity, and head-injured children had a greater 
number of behavioral problems at 1 year follow-up when compared with a randomly selected sample of children, ages 6 to 16 years. (Greenspan AI, MacKenzie EJ. Functional outcome after pediatric head injury. Pediatrics October 1994;94:425-432). (Reprints: Dr Arlene I Greenspan, National Center for Injury Prevention and Control, Centers for Disease Control and Prevention, 4770 Buford Highway, NE, Mailstop F-41, Chamblee, GA 30341).

COMMENT. In children who sustain head injury, the severity of the injury is correlated with preinjury health status, and even those with minor injuries have a greater incidence of chronic health problems than the general population. The risk of post-injury functional limitations was increased in children with a history of chronic illhealth and those who sustained lower extremity injuries. Evidence of cognitive, physical, or behavioral dysfunction requires intervention and rehabilitation.

The frequency of postconcussive symptoms in 41 children (ages 6-12) with traumatic brain injury (TBI) was evaluated at the Dept of Psychology, Case Western Reserve University, Cleveland, OH. Compared to 40 controls with orthopedic injuries only, those with TBI had more postconcussive symptoms both at baseline and at 6 month follow-up. The number of symptoms was related to injury severity and initial loss of consciousness (GCS <9), Nonverbal IQ and the baseline Children's Depression Inventory. (Barry CT, Klein SK, Taylor HG. Validity of postconcussive symptoms in children with traumatic brain injury. Ann Neurol Sept 1994;36:519 [abstr]).

\section{EPIDURAL HEMATOMA ACCIDENTAL INJURY}

A 7 -month-old infant who suffered a fall from a washing machine and sustained a frontal epidural hematoma is reported from the Division of Neurosurgery, Oregon Health Sciences University. The infant had been placed on an engaged washing machine strapped in his car seat and left unattended. Within 2 hours of the fall, the infant became more irritable, vomited, was listless, and had a generalized seizure. CT showed a fracture of the skull and a epidural hematoma. He was discharged on postoperative day 3 , and his development at 13 month of age was normal. Awareness of the potential consequences of this apparently popular and physician-endorsed childconsoling practice is important for physicians who must distinguish accident from abuse. (Hulka F, Piatt J. An infant in a car seat on a washing machine: epidural hematoma. Pediatrics October 1994;94:556-557).

COMMENT. Placement of infants in car seats on vibrating elevated surfaces might have a desired soporific effect but the practice carries the risk of falls and serious head injury. More than two-thirds of brain injuries in infants are attributable to falls, and epidural hematomas occur in 3\%. (Ped Neur Briefs Sept 1992). The rule that children falling from elevated household surfaces do not sustain serious injuries does not apply when an infant is buckled into a car seat. Righting reflexes are vitiated and the weight of the car seat adds to the impact.

Brain injury due to amniocentesis is reported in 4 children with hemiparesis and porencephalic cysts evaluated at Bowman Gray School 